Rapport - Société canadienne d'histoire de l'Église catholique

\title{
Le Curé Louis Nau (fl. 1799-1843)
}

\section{Jean-Jacques Lefebvre}

Volume 24, 1956-1957

URI : https://id.erudit.org/iderudit/1007434ar

DOI : https://doi.org/10.7202/1007434ar

Aller au sommaire du numéro

Éditeur(s)

La Société canadienne d'histoire de l'Église catholique

ISSN

0318-6148 (imprimé)

1927-7075 (numérique)

Découvrir la revue

Citer cet article

Lefebvre, J.-J. (1956). Le Curé Louis Nau (fl. 1799-1843). Rapport - Société

canadienne d'histoire de l'Église catholique, 24, 65-90.

https://doi.org/10.7202/1007434ar

Tous droits réservés @ La Société canadienne d'histoire de l'Église catholique, 1958
Ce document est protégé par la loi sur le droit d'auteur. L'utilisation des services d’Érudit (y compris la reproduction) est assujettie à sa politique d'utilisation que vous pouvez consulter en ligne.

https://apropos.erudit.org/fr/usagers/politique-dutilisation/ 


\section{Le Curé Louis Nau ( $\boldsymbol{f}$. . 1799-1843)}

Il y a plus de dix années, je préparais la première communication que j'eus l'honneur de donner à cette Société, sur la vie religieuse des deux paroisses de mon enfance, Saint-Constant et Saint-Philippe de Laprairie ${ }^{1}$. Quand je me présentai à la chancellerie de l'évêché de SaintJean pour y consulter les archives, l'évêque fondateur du diocèse, $\mathbf{M}^{\mathrm{gr}}$ Anastase Forget, m'invitant à déjeuner à sa table, me fit une observation non dénuée d'humeur : « Je ne vous aime pas, vous autres, historiens, vous n'avez à nous raconter que l'histoire des curés en révolte contre leur évêque. *

Prononcé par un excellent historien lui-même, qui nous a laissé le plus bel ouvrage peut-être sur l'histoire d'un collège, nommément celui qu'il dirigea longuement et avec tant de talent, à l'Assomption, le mot était plein de saveur.

Je savais l'éminent prélat déjà malade et d'une sensibilité que l'on m'avait dit extrême, et je n'eus pas le courage de lui rappeler le mot du grand pape, le saint pontife Léon XIII, qui ouvrit toutes grandes les portes des Archives du Vatican, hermétiquement closes depuis tant de siècles, en rappelant que « si les Evangiles étaient écrits de nos jours, on y tairait le reniement de saint Pierre ", et je me contentai de sourire.

En compilant le monumental Dictionnaire biographique du Clergé canadien-français, du tenace chercheur et habile portraitiste, M. J.-B.-A. Allaire, qui, hélas, n'a pas été relevé depuis son décès, j'avais été naturellement frappé par le manque de précisions de quelques-unes des notices, alors que d'autres sont présentées dans tout l'éclat d'un profil de médaille.

Ainsi, M. Allaire n'a pas mis sous le boisseau les carrières bousculées d'un Chartier de Lotbinière ${ }^{2}$ - le frère du grand ingénieur de la Nouvelle-France - d'abord marié, puis entré dans les Ordres, devenu Récollet, puis interdit et suspendu, passé chez les Cordeliers, « apostat en Europe pendant deux ans », chevalier de Malte, chassé de la Martinique à cause de ses désordres, devenu curé de Saint-Laurent, Ile-d'Orléans, pour deux ans, et de nouveau interdit par son cousin germain, l'évêque de Québec, $M^{g r}$ d'Esglis. Ce que n'a pas connu M. Allaire, ce curieux personnage eut la singulière fortune de devenir

1 V. Rapport 1945-1946, la Société Canadienne dHistoire de IEglise catholique, Imprimerie Leclerc, Hull, P.Q., s.d., p. 125-158.

2 J.-B.-A. Allaire, Dictionnaire biographique du Clergé canadien-français, Montréal, 1910, p. 121, 142, 351, 402, 511, 513. 
le premier aumônier catholique des troupes américaines ${ }^{3}$, dites alors les Insurgés, pendant que son évêque, $M^{\mathrm{gr}}$ Briand, fulminait les interdits et les excommunications pour celles de ses ouailles qui sympathisaient avec les envahisseurs yankees de la province.

M. Allaire ne fait pas mystère ${ }^{2}$ non plus du sort :

- d'un Louis Normandeau (1812-1891), natif de Québec, qui " apostasie et devient ministre baptiste ${ }^{2}$ " quatre ans après son ordination (1835);

- de Hubert-Joseph Tétreau ( $f$. 1803-1878), natif de Verchères, ordonné en 1826, curé de Beauharnois en 1830, des Eboulements en 1838 et qui passe au protestantisme, à Granby, en 1843;

- d'un Ephrem Thérien ( $f$. 1825-1890), ordonné en 1849, d'abord vicaire à Vaudreuil, premier curé de Sainte-Adèle [de Terrebonne], 1852-1855, vicaire à Sainte-Martine [de Châteauguay], 1860, qui - apostasie ${ }^{2}$ et part pour les Etats-Unis en 1863, puis retiré à Granby où il est décédé après un retour à Dieu aux derniers jours de sa vie... \;

- d'un Joseph Dallaire (1813-1893), originaire de Saint-Ours, ordonné en 1838, curé (1838) de Saint-Chrysostome [de Châteauguay], de Rigaud en 1845, d'Henryville en 1848, de Farnham en 1850, de Lacolle en 1852-1853; « apostasie ${ }^{2}$ et fixé à Plattsburg, New-York »;

- sans compter le plus connu d'eux tous, Chiniquy, \& le plus sinistrement célèbre des apostats canadiens ${ }^{2}$, comme dit M. Allaire et à qui M. Marcel Trudel vient de consacrer un magistral ouvrage, et tutti quanti.

Est-il besoin de l'ajouter, il n'y aura pas lieu ici d'évoquer une aussi malheureuse carrière. Les derniers documents qui nous sont parvenus sur la vie du curé Nau témoignent que ses mœurs étaient irréprochables et qu'il semble, à la fin, être rentré dans le giron de l'Eglise.

Louis Nau, né à Lanoraie en 1799 - M. Allaire ne dit où ${ }^{4}-$ ordonné prêtre en 1829 , à l'âge de trente ans, vicaire à Saint-Jacques-

3 Laval Laurent, Québec et l'Eglise aux Etats-Unis sous $M^{\text {or }}$ Briand et $M^{\text {or }}$ Plessis, Montréal, 1945.

4 Au congrès tenu au Collège Loyola, M. l'abbé Hector Geoffroy, du Séminaire de Joliette, m'apprit qu'il devait être de Berthier, son père ayant été l'un des premiers habitants de Sainte-Elisabeth de Joliette. L'acte de baptême de Louis Nau (note de M. J.-Arthur Lebœuf), inscrit le 16 septembre 1799 aux registres de Berthier, indique que ses parents, Charles Nau [l'officiant écrit Naud] et Louise Pagé, étaient de Lanoraie. Ils avaient contracté mariage à Berthier, le 5 juillet 1782 (note de $\mathrm{M}^{\text {me }}$ Florence Fernet-Martel). Charles Nau, le père du curé, était né à Deschambault (Portneuf) à la Noël 1760, du mariage de Joseph Nau et de Marie-Joseph Detailly (C. Tanguay, Dictionnaire généalogi. que..., VI, 139). Le curé Nau signa toujours : Nau. 
de-l'Achigan la même année, à Maskinongé l'année suivante, à SaintHyacinthe l'année d'après, fut, dit M. Allaire, deux ans curé de Rigaud (1832-1834), puis deux ans curé de Saint-Jean-Baptiste-de-Rouville (1834-1836) et finalement serait passé aux Etats-Unis.

Une symphonie inachevée, quoi. Mais, nous allons le voir, le mot symphonie serait de l'antiphrase.

Un autre chercheur infatigable, l'abbé Ivanhoë Caron, le patient annaliste de la Colonisation de la Province de Québec - un magistral ouvrage - survivra peut-être davantage par son analyse, d'intérêt plus général, de la correspondance des évêques de Québec, publiée dans le Rapport de l'Archiviste de la Province de Québec, des années 1927 à 1941, et continuée depuis, pour ce qui a trait à Montréal, par l'abbé Adélard Desrosiers.

Cette savante compilation est comme un miroir de la vie paroissiale de nos aieux, des luttes de nos vieux curés, des difficultés de nos anciens évêques, une source incomparable de sujets d'études pour les monographies qui manquent tant encore pour étayer la grande histoire.

J'avais incidemment vu des allusions en nos vieux recueils de jurisprudence au procès Nau, mais toujours de façon imprécise, encore qu'Edmond Lareau, en son Histoire - non encore dépassée - du Droit canadien soit assez explicite.

On sait que l'élection de $\mathrm{M}^{\mathrm{gr}}$ Lartigue au siège épiscopal de Montréal fut laborieuse entre toutes. Mal agréé de ses confrères sulpiciens ${ }^{5}$, selon les apparences, imparfaitement soutenu du Siège de Québec, non admis du gouvernement de la métropole, à Londres, de juridiction incertaine et mal définie, son autorité mise en doute par des curés du " district 》 de Montréal, on a peine aujourd'hui à se faire l'idée des difficultés sans nombre qui surgirent sur les pas mal assurés de notre premier pontife.

Des curés quasi inamovibles, vieillis sous le harnais en leurs grandes paroisses de l'époque, souvent des autodidactes, formés au hasard de lectures incomplètes, encore sous le coup des impressions des bouleversements sociaux et moraux qu'avaient amenés la Révolution française, et de l'incroyable - de nos jours - décomposition disciplinaire du bas clergé français ${ }^{6}$, s'étaient mis à méditer les vieilles doctrines du gallicanisme et à en extraire des théories ou des opinions qui cadraient avec leurs conceptions, leur orgueil ou... leur intérêt.

Avant la diffusion de l'imprimé, comme nous le connaissons aujourd'hui, et l'instantané des communications dans lequel nous vivons et qui n'est pas loin de créer, chez tant de gens, une confusion mentale dont nous déplorons tous les jours les effets, il semble que l'ouvrage que l'on trouvait le plus souvent dans les bibliothèques ecclésiastiques de

5 Rapport de l'Archiviste de la Province de Québec (ci-après : R.A.P.Q.) pour 1942-1943, p. 163.

6 Pierre de La Gorce, Histoire religieuse de la Révolution française, Paris, 1912-1923. 
l'époque était le vieil historien et canoniste gallican du grand siècle, Fleury ${ }^{7}$, un nom démonétisé de nos jours, mais une grande autorité des temps passés.

Il faut savoir encore que du côté laïque, les œuvres des encyclopédistes - que le marquis de Montcalm lisait au moment de leur publication entre deux campagnes de la guerre de Sept ans - n'avaient jamais cessé de circuler dans la province, comme s'en plaignit amèrement $\mathbf{M}^{\text {sF }}$ Hubert, évêque de Québec ${ }^{8}$, surtout après l'importation des livres français autorisée du temps de Haldimand.

Dans les années 1820-1830, quelques curés de la région de Montréal dressèrent une campagne sourde de résistance contre le nouvel évêque élu de Montréal, et c'est ainsi que nous avons de petits pamphlets anonymes, ou publiés par des prête-nom, par le riche curé Augustin Chaboillez, de Longueuil ${ }^{9}$, l'intrigant et orgueilleux François-Xavier Pigeon, le non moins riche curé de Saint-Philippe-de-Laprairie ${ }^{9}$.

Participèrent encore à cette lutte, en cette lointaine époque de doctrine encore mal assurée, J.-B. [Gauthier-] Saint-Germain (17881863) originaire de Boucherville, d'abord vicaire (1811) à Notre-Dame de Montréal, curé de Terrebonne (1818) pendant dix ans, puis de SaintLaurent près Montréal ici plus de trente ans (1829-1863). Il devait s'amender plus tard, et faire ouvre apostolique en fondant le collège, devenu la grande institution que nous connaissons à Saint-Laurent. Et encore, Ignace-Raphaël Leclerc (1767-1833), curé de Saint-Laurent (1817), avant Saint-Germain, dès lors retiré à Montréal et qui menait la campagne, dont ses autorités étaient loin d'être satisfaites ${ }^{9}$.

Cette longue introduction n'a pour but que d'éclairer l'atmosphère qui prévalait dans les milieux ecclésiastiques de la vallée du SaintLaurent au premier tiers du siècle dernier, comme peut se le représenter un profane, qui est loin d'être au fait de l'évolution, génération après génération, de toutes les modalités du droit ecclésiastique, même en notre seul pays.

Il peut paraître singulier que l'abbé Nau, une fois ordonné, ne semblât faire qu'un an ou deux aux diverses paroisses et cures où il passa en sa courte existence connue.

Cela devient moins étonnant lorsque l'on observe que son évêque, dès l'époque de sa première nomination, prend soin de noter dans son avis au curé que le nouveau desservant a demande d'être surveillé ${ }^{10}$ *.

Un an plus tard, mois pour mois, son curé, l'abbé Louis Marcoux, de Maskinongé, rapporte qu'il a plusieurs plaintes contre lui et dit qu'il aimerait mieux "s'en passer que d'en avoir un de cette trempe ${ }^{11}$.

7 Fleury [Claude] (1640-1732).

8 R.A.P.Q. pour 1930-1931.

9 R.A.P.Q. pour 1941-1942, p. 402.

10 R.A.P.Q. pour 1934-1935, p. 369.

11 R.A.P.Q., passin, p. 420. 
Quinze jours se passent et l'évêque, $M^{\text {gr }}$ Panet, lui retire ses pouvoirs comme vicaire à Maskinongé parce que, au rapport du grand vicaire, M. Cadieux, Nau s'est conduit « envers le curé et ses paroissiens d'une manière fort impudente ${ }^{12}$ \. $M^{\text {gr }}$ Lartigue, d'autre part, estime que l'affaire a fait trop de bruit et qu'il ne s'agissait que d'incompatibilité d'humeur entre le vicaire et le curé ${ }^{13}$.

En octobre 1831, son Ordinaire le nomme desservant à la cure nouvellement ouverte de Saint-Cyprien de Napierville. En fait, c'est M. Laurent Amiot - le fils du bel orfèvre de Québec - qui y fut envoyé. Il y fut fort malheureux, en particulier pendant le camp de Napierville de novembre 1838, qui vit près de trois mille hommes sous les armes, alors que les Patriotes occupèrent le presbytère et tinrent le curé comme une sorte d'otage.

En mars 1832, Louis Nau recevait, avec juridiction sur la seigneurie d'Argenteuil, la cure de Sainte-Madeleine de Rigaud ${ }^{14}$. Il y succédait à messire Hyacinthe Hudon, plus tard (1845) vicaire général de Montréal et qui fut emporté par l'épidémie de typhus de 1847.

La calligraphie de M. Nau aux doubles des registres de Rigaud, déposés aux archives de Montréal, est révélatrice de son caractère. Il lui fallait parfois une page entière pour $\mathrm{y}$ inscrire deux simples actes de naissance et de décès ( $V$. le 15 juin 1832), alors que son prédécesseur en inscrivait habituellement quatre, et encore une page entière pour un acte de mariage (V. le 10 janvier 1834).

Dès janvier $1833, \mathrm{M}^{\mathrm{gr}}$ Lartigue conseille au curé Nau, de Rigaud, de retirer sa poursuite contre J.-B. Bédard, qui a refusé d'acquitter les honoraires de la célébration de son mariage ${ }^{15}$.

Un mois plus tard, l'évêque écrit encore au curé qu'il refuse de répondre à sa lettre parce qu'il n'a pas tenu compte de son avis dans l'affaire Bédard et à cause de la vingtaine de procès intentés à ses paroissiens ${ }^{16}$.

La même année, $M^{g r}$ Lartigue, écrivant à l'évêque de Québec, $M^{\text {gr }}$ Signay, regrette que M. Nau soit resté à Rigaud et que celui-ci menace de se justifier dans les journaux ${ }^{17}$.

Cependant en novembre suivant, l'évêque étend les pouvoirs du curé Nau aux cantons de Chatham, Granville, Wentworth (Argenteuil) ${ }^{18}$.

En décembre 1833, $\mathrm{M}^{\mathrm{gr}}$ Lartigue, découragé, écrit à son supérieur de Québec : *Il faut laisser M. Nau se tirer d'embarras seul, il n'y a

R.A.P.Q. pour 1935-1936, p. 137.

R.A.P.Q. pour 1942-1943, p. 96.

R.A.P.Q., passim, p. 135.

R.A.P.Q. pour 1943-1944, p. 213.

R.A.P.Q., passim, p. 217.

R.A.P.Q., passim, p. 240.

R.A.P.Q., passim, p. 246. 


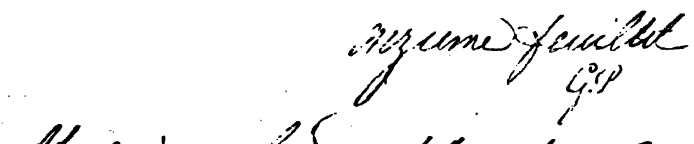

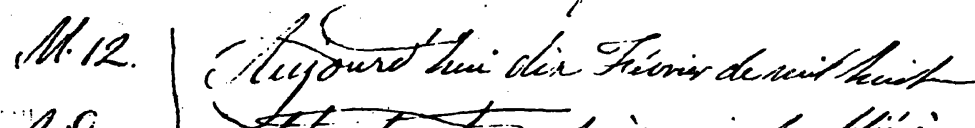

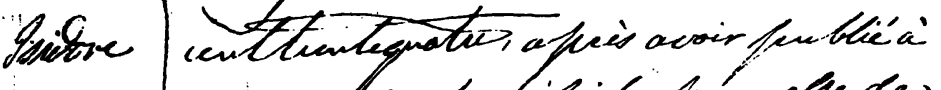

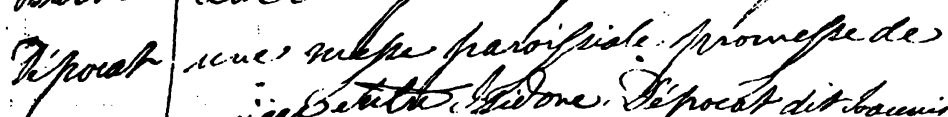

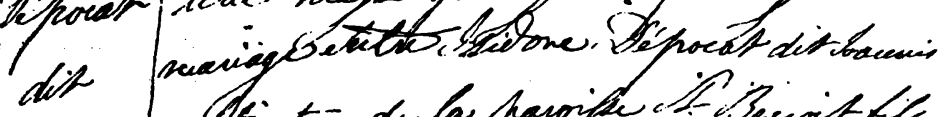

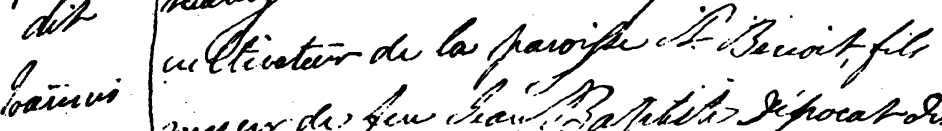

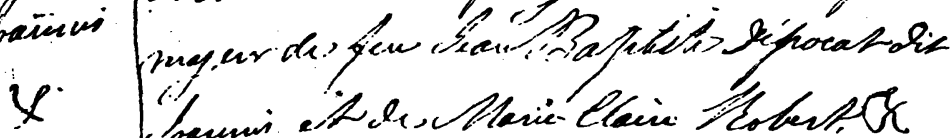

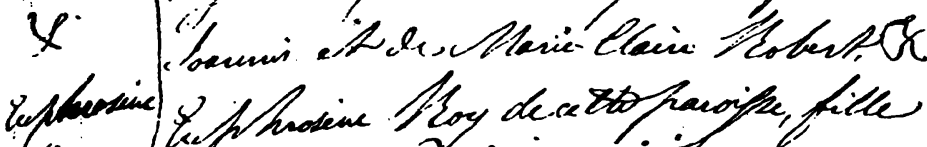

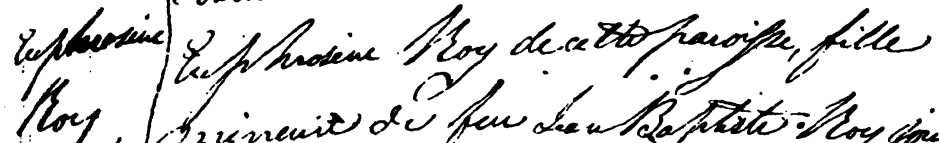

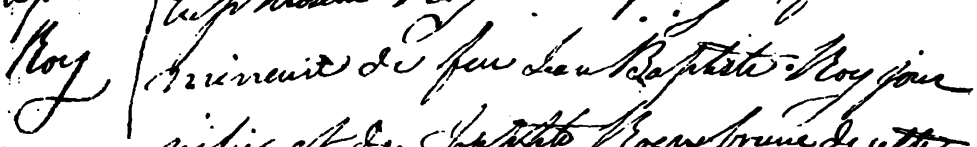

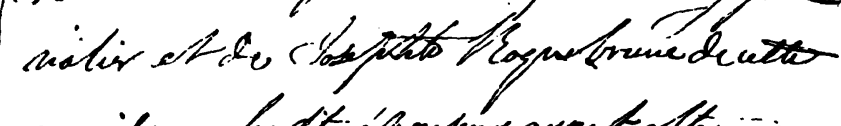

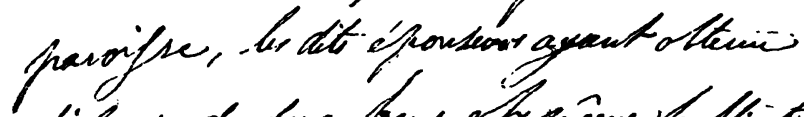

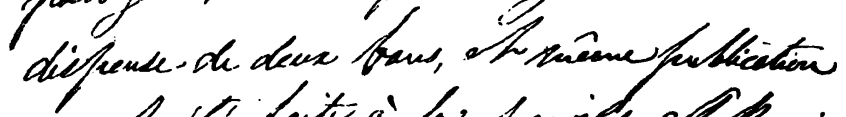

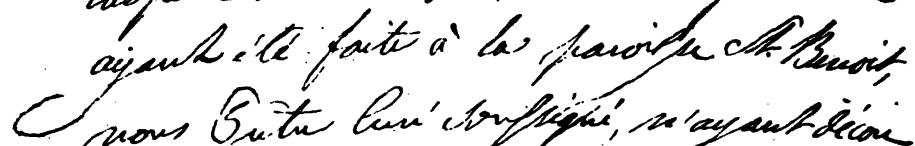

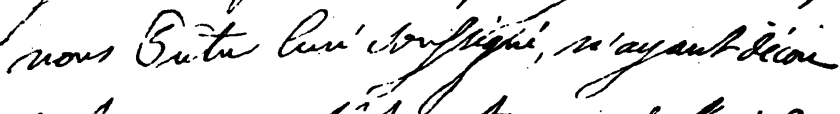

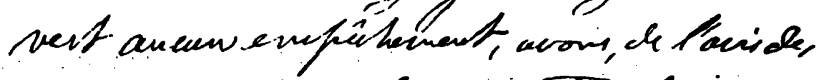

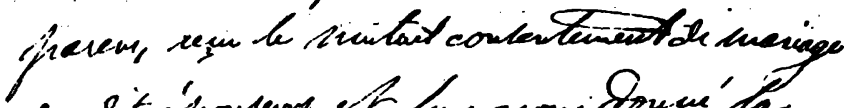

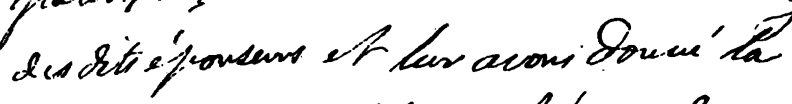

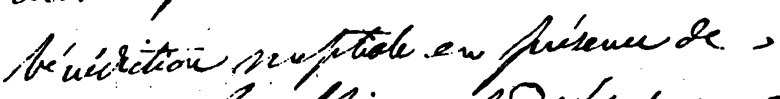

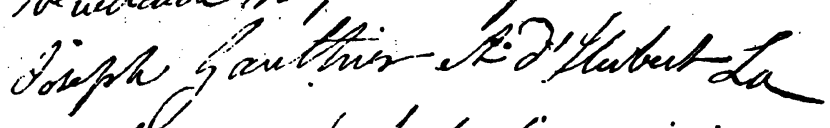

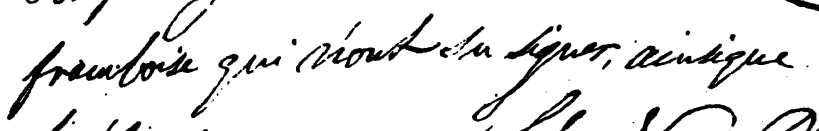
lesefioux..

clo laube

$-70-$ 
rien à faire avec lui, et s'il quitte la paroisse il devrait aussi sortir du district ${ }^{19}$.

$\mathrm{M}^{\mathrm{gr}}$ Signay - un diplomate, qui passa son temps à raccorder des mutins - prévient le curé de Rigaud que a pour ramener la tranquillité dans la paroisse... il sera obligé de le nommer à une autre... où il pourra travailler avec plus de succès ». En janvier 1834, il le nomme à la cure de Saint-Jean-Baptiste de Rouville, paroisse voisine de Saint-Hyacinthe où M. Nau avait été vicaire en 1831-1832. Il y était appelé à succéder à M. Pierre Lafrance, qui passait à Rivières-des-Prairies.

$M^{\text {gr }}$ Lartigue prévient $M^{\text {gr }}$ Signay, en mars 1834, que $M$. Nau s'entête à ne pas quitter sa cure de Rigaud. Enfin il lui fait rapport, en mại 1834, qu'il est rendu à Saint-Jean-Baptiste de Rouville ${ }^{20}$.

Le nom de M. Nau apparaît, en septembre 1835, à la liste - mais tout au bas - du clergé de Montréal qui adressait une supplique à l'archevêque de Québec demandant un siège épiscopal à Montréal.

$M^{g r}$ Lartigue écrivant à M. Nau, à Saint-Jean-Baptiste, en novembre 1835 , lui demande de ne pas paraître triompher publiquement de la réponse qu'il lui a donnée touchant les plaintes déjà formulées par ses paroissiens, MM. Coursol et de Rouville ${ }^{21}$.

Février $1836: \mathrm{M}^{\mathrm{gr}}$ Lartigue réfère à l'évêque de Québec une requête des habitants de Saint-Jean-Baptiste (de Rouville) qui se plaignent de leur curé (Nau) pour ses injures envers eux et envers leur seigneur M. de Rouville.

Un mois plus tard, il saisit encore $M^{\text {gr }}$ Signay de l'affaire Nau, se disant convaincu que ce dernier ne peut tenir en sa paroisse où on l'a pendu en effigie, comme il l'avait été à Rigaud ${ }^{22}$.

En mars 1836, M. Nau n'a pas encore fait deux ans à Saint-JeanBaptiste que l'archevêque de Québec prévient son auxiliaire, l'évêque de Telmesse, non encore intronisé évêque de Montréal, de prendre les procédures nécessaires contre $M$. Nau, en ajoutant que a ce n'est pas le temps de porter cette affaire devant les tribunaux > et, en juin suivant; il écrit au curé pour le prévenir qu'à l'automne il sera transféré à une autre cure.

Pourtant l'évêque hésite à intervenir ${ }^{23}$.

Les événements vont se précipiter.

Le 9 juillet 1836, $\mathrm{M}^{\mathrm{gr}}$ Lartigue avise le curé de Saint-Jean-Baptiste qu'il a reçu une requête de quarante habitants qui se plaignent d'avoir été atrocement insultés en chaire. Il l'invite a pour votre honneur et

10 R.A.P.Q., passim, p. 252.

20 R.A.P.Q., passim, p. 264.

21 R.A.P.Q., passim, p. 321.

22 R.A.P.Q. pour 1944-1945, p. 183, 184

23 R.A.P.Q., passim, p. 185. 
celui du clergé, à résigner. $M^{g r}$ Lartigue en réfère à l'évêque de Québec ${ }^{24}$ à qui il recommande de placer Nau à Saint-Valentin, où il pourrait être utile à cause de son anglais ${ }^{25}$.

L'agitation politique battait alors son plein par toute la province, les assemblées politiques se multipliaient, les journaux chauffaient l'opinion à blanc. Aux esprits avertis, les signes avant-coureurs de la levée d'armes étaient déjà visibles.

$M^{\mathrm{gr}}$ Lartigue implore encore $\mathrm{M}^{\mathrm{gr}}$ Signay, en septembre 1836 , de régler le cas de $\mathrm{M}$. Nau, et lui relate que celui-ci a pris possession, par acte devant notaire, de la cure de Saint-Jean-Baptiste ${ }^{26}$.

Le même mois, $M^{\mathrm{gr}}$ Lartigue écrit à $M$. Nau qu'il a accompli l'acte le plus ouvert de révolte contre l'Eglise, en prenant possession, en titre, d'une cure qu'il avait reçue révocable à volonté de l'évêque.

- Vous n'aurez plus juridiction dans la paroisse, ajoute-t-il, et aucune juridiction dans tout le diocèse ${ }^{27}$.

Le même mois, il lui enjoint d'évacuer le presbytère de la paroisse, de remettre à la garde du marguillier en charge tout ce qui appartient à l'église : registres, comptes, vases sacrés.

A la même date, il rappelle de Rivière-des-Prairies M. Lafrance, ancien curé de Saint-Jean-Baptiste, et le nomme à nouveau en son ancienne paroisse, en lui recommandant de demander l'appui du seigneur, $M$. de Rouville, et il lui indique la procédure pour se faire céder le presbytère. En octobre suivant, il revient sur le sujet dans ses instructions à $M$. Lafrance, et lui enjoint de sommer le curé, qui n'a pas quitté le presbytère, devant deux témoins ${ }^{28}$.

Et l'interdit part, le même mois, pour le curé Nau.

On a dit que les juristes, bien avant les grammairiens et les littérateurs, ont donné sa structure à la syntaxe française. On en verra un exemple encore ici dans cette injonction, si terrible dans sa teneur. Citons ce texte au mot.

Monsieur,

Montréal le 25 octobre 1836

Vous êtes accusé devant mon Tribunal d'avoir refusé, après plusieurs sommations de livrer au Curé nouveau de St Jean Baptiste, Mr Lafrance et aux Marguillers de cette Paroisse l'église, la sacristie, le cimetière et les choses y appartenantes, ainsi que le Presbytère et ses dépendances, contrairement aux ordres que je vous avais signifiés là dessus : d'avoir refusé au nouveau curé sus-dit l'usage de ces divers lieux et choses y appartenantes, pour y faire les fonctions Sacerdotales et curiales : que vous avez essayé de déléguer pour faire un mariage de votre paroisse,

24 R.A.P.Q., passim, p. 194, 195.

25 R.A.P.Q., passim, p. 200.

26 R.A.P.Q., passim, p. 205.

27 R.A.P.Q., passim, p. 209.

28 R.A.P.Q., passim, p. 212. 
un pouvoir que vous reconnaissiez n'avoir pas vous-même; que vous avez refusé de livrer à Mr Lafrance la boîte à l'huile des infirmes, pour secourir une femme réputée dangereusement malade, ne pouvant ou ne voulant aller vous-même à son secours : d'avoir refusé, contre mes ordres, de consommer les Stes Espèces de l'Eucharistie qui se trouvoient alors dans l'Eglise de St Jean-Baptiste. C'est pourquoi, par la présente, je vous somme et enjoins de comparoitre personnellement devant moi, dans ma maison de St Jacques à Montréal, jeudi le troisième jour de novembre prochain à dix heures du matin, pour répondre aux chefs d'accusation sus-dits et recevoir mon jugement sur iceux.

J. J., Evêque de Montréal 29.

Et $M^{\text {er }}$ Lartigue charge $M$. Prince, directeur du Collège de SaintHyacinthe, de la signification de l'injonction ${ }^{30}$.

En octobre 1836, $\mathrm{M}^{\mathrm{gr}}$ Lartigue, écrivant à $M$. Lafrance à Saint-JeanBaptiste, à qui il avait déjà donné en septembre des instructions sur la procédure pour prendre possession de la cure, lui écrit encore de sommer M. Nau devant deux témoins et il lui recommande de rallier tous les paroissiens autour de lui et de ne pas céder un pouce de terrain ${ }^{31}$.

Après un semblant de pacification, écrivant au curé Lafrance, l'évêque permet, en novembre 1836, à M. Nau d'assister aux offices, dans le chœur, en surplis ${ }^{32}$.

Mais la bataille n'est pas close quant à la garde des registres des actes de baptême, mariage et sépulture, et, en décembre 1836, l'évêque donnait avis au protonotaire de Montréal que Nau n'était plus curé de Saint-Jean-Baptiste depuis le 27 septembre et qu'il n'avait fait aucune fonction sacerdotale depuis le 23 novembre ${ }^{33}$.

En novembre 1836, l'évêque de Montréal sollicitait de l'archevêque de Québec des renseignements précis sur la fixation des cures avant la conquête ainsi que copie de la lettre du ministre Maurepas à $\mathbf{M}^{\mathrm{gr}}$ de Pontbriand, en avril $174.1^{34}$.

Il lui recommandait encore « d'empêcher le Canadien de publier des erreurs sur l'affaire Nau, comme dans ses numéros des 18 et 21 novembre, erreurs d'ailleurs rectifiées par la Minerve du 24 ..

Encore en décembre 1836, il autorisait le curé Lafrance, de SaintJean-Baptiste, de prendre « l'argent du coffre » pour les frais de l'avocat et des syndics dans le procès de l'ex-curé Nau.

Visiblement, le curé Nau, renonçant à ses immunités ecclésiastiques, était sur le point d'en appeler à la justice civile.

Archives de l'Archevêché de Montréal. Registres, Correspondance de M"r Lartigue.

30 R.A.P.Q. pour 1944-1945, p. 215.

31 R.A.P.Q., passim, p. 212.

32 R.A.P.Q., passim, p. 218.

33 R.A.P.Q., passim, p. 221.

34 R.A.P.Q., passim, p. 223. 


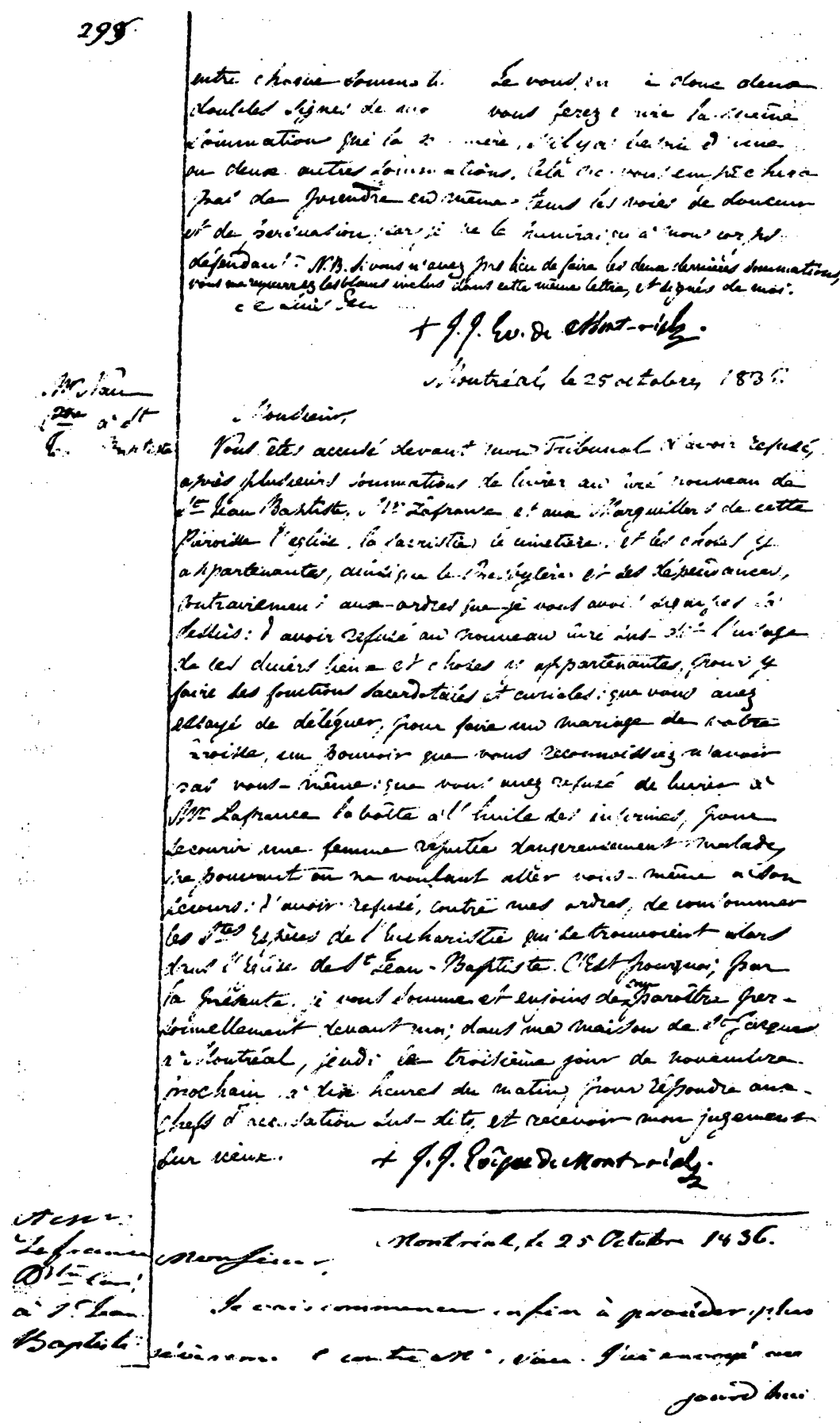


Les dossiers sont conservés aux archives du palais de justice de Montréal des deux causes inscrites en février 1837 à la Cour du Banc du roi - tribunal principal de Montréal avant la constitution de la Cour supérieure en 1849 - par le curé Nau, l'une contre son successeur désigné à Saint-Jean-Baptiste, M. Pierre Lafrance, et l'autre contre son évêque, $\mathbf{M}^{\text {gr }}$ Lartigue $^{3 \tilde{}}$.

M. Nau avait confié ses intérêts à l'étude d'avocats La Fontaine et Berthelot, formée, comme l'on sait, du futur sir Louis-Hippolyte La Fontaine, le grand artisan de notre évolution constitutionnelle aux lendemains de 1840, et qui fut, selon un témoignage recueilli de l'un de ses proches dans la magistrature, beaucoup plus un libéral, au sens doctrinaire du mot, qu'il ne voulut, ou sut ne le laisser paraître de son vivant, et de son beau-frère, Amable Berthelot, devenu, lui aussi, juge à la Cour supérieure.

L'intérêt de ces causes réside surtout dans les déclarations produites par les avocats.

On y voit de quoi retournait la pensée juridique, canonique ou civile, de ce temps. Quant au style, inutile d'ajouter qu'il est de l'époque. Mais à voir certains mémoires ou arrêts des tribunaux, même certains textes législatifs de nos jours, peut-on vraiment se flatter d'avoir tellement progressé ?

District de

Montréal

\section{COUR DU BANC DU ROI}

$\begin{array}{lr}\text { Messire Louis Naud } & \text { Dem }^{\text {vs }} \\ \text { Messire Pierre Lafrance } & \text { Def. }\end{array}$

Messire Louis Nau, prêtre \& curé de la paroisse de $S^{t}$ Jean Baptiste de Rouville, dans le District de Montréal, et résident en la dite paroisse, Demand $^{r}$ contre Messire Pierre Lafrance, Prêtre, ci-devant résident en la paroisse de la Rivière des Prairies, dans le dit District, et maintenant résident en la dite paroisse de $\mathbf{S}^{\mathbf{t}}$ Jean Baptiste de Rouville, Défen ${ }^{\mathbf{d}}$

Déclare :

Que led ${ }^{t}$ Demd $^{r}$ est sujet britannique, né en cette Province, \& y a toujours résidé.

Que depuis plusieurs années et notamment depuis dès avant 1834, le dit Demand ${ }^{r}$ est Prêtre séculier duement ordonné comme tel en cette Province, Diocèse de Quebec, et que depuis l'époque de son ordination susdite antérieure à la dite année 1834, il a toujours eu, comme, Prêtre séculier, les qualités et capacités nécessaires et requises pour desservir \& posséder, conformément aux loix du Pays, aucune cure ou Bénéfice en cette Province.

Qu'au commencement de la dite année 1834, la dit Défendr était curé de la dite paroisse de $\mathrm{S}^{\boldsymbol{t}}$ Jean Baptiste : Mais que vers le commencement du mois d'avril de la même année, la dite cure devint vacante

35 Rég. C.B.R. [Cour du Banc de la Reine], Montréal, 1838, vol. II, p. 1577-1581. M. l'abbé Honorius Provost, président (1957) de la Société, a savamment traité de l'aspect juridique de cette question du Régime des Cures..., dans le Rapport de cette Société pour 1954-1955, pp. 85-103. 
par la démission abandon, ou résignation qu'en fit, vers cette époque, le dit Defendeur, qui dès lors fut pourvu \& nommé à la cure de la dite paroisse de la Rivière des Prairies.

Que le dit Demandeur fut, de son propre consentement, duement canoniquement et légalement pourvu et nommé à la desserte, comme curé, de la dite cure et paroisse de $S^{t}$ Jean Baptiste de Rouville, par Sa Grandeur Monseigneur Joseph Signay, Evêque en titre du dit Diocèse de Quebec dont dependait la dite cure ou bénéfice, et ce en vertu de différentes Lettres, titres ou provisions, et plus particulièrement par des Lettres, titres ou Provisions en date du 8 Avril 1834, signées et octroyées librement par le dit Sieur Evêque en faveur du dit Demandeur, et dont l'effet était et a été de rendre, établir et instituer ce dernier curé ou benéficier à tous égards, de la dite cure et paroisse de $\mathbf{S}^{\mathbf{t}}$ Jean Baptiste de Rouville, pour par lui en avoir seul comme tel la legitime possession, et aussi jouir de tous les droits, privilèges, fruits \& revenus y attachés, en vertu et en conformité des loix du pays.

Que la nomination dud ${ }^{t}$ Demandeur à la dite cure et benefice de la dite paroisse de $\mathbf{S}^{\mathbf{t}}$ Jean Baptiste de Rouville, fut ainsi faite comme sus dit par celui qui en avait le pouvoir légal, savoir le dit Sieur Evêque de Quebec, en vertu de la loi du pays, et plus particulièrement en vertu de l'arrêt du conseil d'Etat du Roi de France, du 27 Avril 1699, duement onregistré (sic) en cette Province, lequel Arret donne a l'Evêque diocésain, privativement à toutes autres personnes, la collation ou patronage des cures ou Bénéfices en ce Pays.

Que le dit Demandeur ayant agrée cette nomination et accepté la collation de la dite cure ou benéfice de la dite paroisse de $\mathrm{S}^{\mathbf{t}}$ Jean Baptiste de Rouville, ainsi faite en sa faveur par le dit Sieur Evêque Diocésain, en exécution des loix du pays, en est par cela même devenu de fait et de plein droit, le seul curé \& desservant legitime; qu'en cette qualite et capacité, et à ce titre, le dit Demand ${ }^{r}$ 's'est mis vers la fin d'avril ou en Mai 1834, en possession réelle et légale de la dite cure ou bénéfice ainsi a lui conféré et alors devenu vacant comme dit est et de leurs circonstances \& dependances : que depuis ce tems là il a toujours seul, comme curé de la dite paroisse de $S^{\mathfrak{t}}$ Jean Baptiste, possédé la dite cure ou bénéfice \& leurs circonstances et dépendances, et joui des droits, privilèges, fruits \& revenus y attachés, et ce légalement, paisiblement, publiquement, et au vu et au sçû de tout le monde, et en particulier du dit Défendeur en cette cause.

Qu'outre cette possession légale, réelle et continue de la dite cure et benéfice pendant plusieurs années, le dit Demandeur a, D'abondant, et pour mieux faire connaitre au public cette possession, ainsi que les droits et privilèges qu'il $y$ avait acquis, et pour en assurer la conservation, fait, le 3 Septembre dernier, acte solennel, authentique et par écrit, de prise de possession civile de la dite cure et bénéfice, ce dont acte solennel, authentique \& légal a été redigé et reçu par écrit pardevant $\mathbf{M}^{\text {tre }}$ Charles Tétu et ses confrères notaires, le dit jour 3 Septembre dernier, lequel acte de prise de possession a été duement insinué et enrégitré (sic) dans le regitre (sic) des insinuations de cette cour, avec, en même tems, les lettres, titres ou provisions susdites accordées et octroyées comme dit est par le dit Sieur Evêque en faveur dud ${ }^{t}$ Demandeur.

Qu'en conséquence de tout ce que dessus, le dit Demd $^{r}$ depuis led ${ }^{t}$ jour ou il a été pourvu et nommé comme curé, à la dite cure ou bénéfice de lad ${ }^{\text {te }}$ paroisse de $S^{t}$ Jean Baptiste, et qu'il a agrée et accepté cette nomination \& qu'il s'est mis en possession réelle \& légale de la dite cure ou bénéfice, a été et est encore le seul curé de la dite paroisse, que comme tel il a possédé la dite cure et bénéfice, y a perçu la dime, y a exercé en autant quil était en son pouvoir et conformément aux loix du pays, les devoirs de curé, a été comme tel, de droit et de fait, le 
possesseur et le gardien du presbytère, de l'Eglise, sacristie \& cimetière de la dite paroisse de $\mathbf{S}^{\mathbf{t}}$ Jean Baptiste, ainsi que de leurs circonstances et dépendances, et des droits \& privilèges y attachés.

Que du moment que led ${ }^{t}$ Demand ${ }^{r}$ a été pourvu et nommé comme susdit, comme curé, à la desserte de la dite cure ou bénéfice \& qu'il a accepté cette collation ainsi faite en sa faveur par led ${ }^{t} S^{r}$ Evêque Diocésain, et qu'il a eu pris comme dit est possession de la dite cure et bénéfice, il devait être, a été et est encore inamovible et perpétuel dans la possession de la dite cure et bénéfice et des droits et privilèges y attachés, et ce en vertu des loix en force en cette Province, et doit y être considéré et déclaré comme tel : et qu'étant seul curé \& desservant legitime de la dite cure et paroisse, aucun autre que lui ne peut y exercer legalement les fonctions curiales, et prendre le titre $i \&$ le nom de curé de la dite paroisse, tant que led. ${ }^{t}$ Demd n'aura pas librement \& de bon gré résigné la dite cure ou benefice, ou qu'il ne s'en sera pas démis librement et de bon gré, ou tant qu'il n'en aura pas été dépossédé et exproprié par un Jugement de cette cour, et que la dite cure ou bénéfice n'aura pas été déclaré vacant par un Jugement de cette cour.

Que nonobstant cette possession et cette jouissance: par le dit Demd ${ }^{r}$, de la dite cure ou bénéfice de la dite paroisse de $S^{t}$ Jean Baptiste de Rouville, et de ses circonstances et dépendances, ainsi que des droits et privilèges y attachés le dit Defendeur qui en avait pleine et entière connaissance, a cependant, au mépris de la loi et des droits acquis du Demd $^{\mathbf{r}}$, puis, dans le cours du Mois de Septembre dernier, et continuellement depuis cette époque jusqu'à ce jour, et prend encore la qualité $\&$ le titre de curé \& de desservant de la dite paroisse de $\mathrm{S}^{\mathbf{t}}$ Jean Baptiste de Rouville, et ce sans autorité capacité \& droit quelconque, mais dans la vue seulement de troubler led ${ }^{t}$ Demd $^{r}$ dans sa légitime possession et jouissance de la dite cure ou bénéfice \& de ses circonstances et dépendances, et de l'en spolier et exproprier : que depuis le ou vers le 26 Septembre dernier, et continuellement depuis cette époque jusqu'à ce jour, le dit Defendeur a pris de force, par violence \& même à main armée, et ce sans aucun droit quelconque possession de la dite Eglise, sacristie $\&$ cimetiere de la dite paroisse de $\mathbf{S}^{\mathbf{t}}$ Jean Baptiste, et des Meubles, effets, vases sacrés et ornemens sacerdotaux qui se trouvaient dans la dite Eglise et sacristie \& qui étaient en la possession et garde légales dudit Demand ${ }^{\mathbf{r}}$, laquelle possession \& detention injuste et illegale led ${ }^{\mathbf{t}}$ Def retient encore : que depuis \& durant la même période jusqu'à ce jour led ${ }^{t}$ Défend ${ }^{\mathbf{r}}$ a pris sur lui d'exercer sans aucun droit $\&$ autorité quelconque, et malgré même les défenses expresses dud ${ }^{t} \mathrm{Demd}^{r}$, les fonctions curiales dans la dite paroisse de $\mathrm{S}^{t}$ Jean Baptiste, où il les exerce encore, et ce au grand préjudice du dit Demandeur.

Que led ${ }^{t}$ Demand ${ }^{r}$, par cette conduite illégale ces voies de fait et violences de la part du Défend ${ }^{r}$, a été depuis le ou vers le 26 Septembre dernier et tous les jours depuis cette époque jusqu'à ce moment, et est encore troublé par led ${ }^{t}$ Def dans sa juste et légitime possession de la dite cure ou benefice de la dite paroisse de $\mathbf{S}^{\mathbf{t}}$ Jean Baptiste, et des dites Eglises, sacristie, presbytere et cimetière et de leurs circonstances \& dépendances, ainsi que dans la jouissance des droits et privilèges que le dit Demd y a acquis : et comme led $^{t}$ Defendeur persiste injustement \& illégalement à troubler \& vexer ainsi led ${ }^{t} \operatorname{Demd}^{r}$, ce dernier se trouve dans la nécessité de se pouvoir (sic) contre lui par une action en complainte devant cette cour.

Qu'en outre le dit Défend ${ }^{r}$, par cette conduite illégale de sa part, a fait éprouver au Demand ${ }^{r}$ des dommages au montant d'au-moins $L 600$ - cours actuel -

Pourquoi le dit Demandeur conclut à ce que pour les causes et considerations ci-dessus mentionnées, les Provisions qui pourraient avoir 
été obtenues par le dit Défendeur, pour desservir la dite cure ou bénéfice de la dite paroisse de $\mathbf{S}^{\mathbf{t}}$ Jean Baptiste de Rouville, soient à tel égard que de raison, déclarées nulles, ensemble ce qui les a suivies et précédées, et que lui le dit Demandeur soit maintenu et gardé en la possession et jouissance de la dite cure ou benéfice et des dites Eglises, sacristie, presbytère et cimetière, ainsi que des droits, privilèges fruits et revenus y attachés, de laquelle cure ou bénéfice led ${ }^{t} \operatorname{Demd}^{r}$ a été comme dit est canoniquement et légalement pourvu : qu'il soit fait défenses au dit Defend $^{\mathrm{r}}$ de troubler led ${ }^{\mathrm{t}}$ Demandeur en la dite possession et jouissance, en quelque manière que ce soit, qualification de titres ou autrement, à ce que led ${ }^{t}$ Defend $^{r}$ soit en même tems condamné à restituer au Demand ${ }^{r}$ les fruits par lui perçus et qu'il percevra par la suite de la dite cure ou bénéfice et de ses circonstances et dépendances : durant son injuste detention, suivant la liquidation qui en sera faite, led ${ }^{t}$ Demd $^{x}$ se reservant le droit de prendre à ce sujet des conclusions ultérieures, s'il devient nécessaire : qu'en cas de contestation sur tout ce que dessus la recréance de la dite cure ou bénéfice et des dites Eglise, sacristie, presbytère \& cimetiere \& de leurs circonstances et dépendances, soit adjugée au Demandeur à sa caution juratoire : et qu'enfin led ${ }^{\mathbf{t}}$ Def soit condamné en outre à payer aud ${ }^{t}$ Demd ${ }^{r}$, par forme de dommages, la somme de $L 600$ cours actuel, sans préjudice aux dommages ultérieurs du dit Demd $^{r}$, si le dit Défendeur persiste dans son injuste détention : Le tout avec intéret et dépens.

Montreal 13 Janvier 1836.

La Fontaine \& Berthelot av $^{\text {ts }}$ du Demd

District de

Montréal

\title{
COUR DU BANC DU ROI
}

\author{
Messire Louis Nau - Dem ${ }^{r}$ \\ vs \\ Mgr J. J. Lartigue - Def
}

Terme de Fevrier 1837.

Messire Louis Nau, Prêtre, et curé de la paroisse de $\mathbf{S}^{t}$ Jean Baptiste de Rouville, dans le District de Montreal, et résident en la dite paroisse, Demandeur contre Sa grandeur MonSeigneur Jean Jacques Lartigue, Evêque de Montréal, résident en la cité de Montreal, dans le dit District de Montreal, Def ${ }^{\mathbf{r}}$

Déclare :

1 Que le dit Demandeur est sujet britannique, né en cette Province et y a toujours résidé.

2 Que depuis plusieurs années et notamment dès avant l'année 1834, le dit Demandeur est Prêtre Séculier duement ordonné comme tel en cette Province, dans le Diocèse de Québec, et que depuis l'époque de son ordination susdite antérieure a $\mathrm{La}$ dite année 1834, il a toujours eu, comme Prêtre Séculier, les qualités et capacités nécessaires et requises pour desservir et posséder, conformément aux loix du pays, aucune cure ou bénéfice en cette Province.

\section{Def en droit}

3 Qu'au commencement de la dite année 1834, Messire Pierre Lafrance, Prêtre, étoit curé de la dite paroisse de $\mathrm{S}^{\mathbf{t}}$ Jean Baptiste, mais que dans le Mois d'Avril ou de Mai de la Même année ou vers ce tems là, la dite cure étant devenue vacante par la démission, abondon ou : resignation 
qu'en fit volontairement vers cette époque le dit Pierre Lafrance qui fut alors pourvu et nommé à la cure de la paroisse de la Rivière des Prairies, le dit Demandeur en cette cause fut de son propre consentement, duement, canoniquement et légalement pourvu et nommé à La desserte, comme curé de la dite cure et paroisse de $\mathbf{S}^{\mathbf{t}}$ Jean Baptiste de Rouville, par Sa grandeur MonSeigneur Joseph Signay, Evêque en titre dud ${ }^{t}$ Diocese de Quebec dont dépendait à cette époque la dite cure ou bénéfice, et ce en vertu de differentes Lettres, titres ou Provisions, et plus particulièrement par des Lettres, titres ou Provisions en date du 8 Avril 1834, signées et octroyées librement par led ${ }^{\mathfrak{t}}$ Sieur Evêque de Quebec en faveur du dit Demandeur, et dont L'effet était et a été de rendre, êtablir et instituer ce dernier curé ou bénéficier à tous égards de la dite cure et paroisse de $\mathbf{S}^{\mathbf{t}}$. Jean Baptiste de Rouville, pour par lui en avoir seul comme tel la légitime possession, et aussi jouir de tous les droits, privilèges, fruits et revenus y attachés, en vertu et en conformité des loix du Pays.

4 Que la susdite nomination du dit Demand ${ }^{r}$ a la dite cure et bénéfice de la dite paroisse de $\mathbf{S}^{t}$ Jean Baptiste de Rouville, fut ainsi faite comme susdit par celui qui en avait le pouvoir legal, Savoir led ${ }^{t}$ Sieur Evêque de Quebec, en vertu de la loi du pays, et plus particulièrement en vertu de l'Arrêt du Conseil d'Etat du Roi de France du 27 Avril 1699, duement enregitre (sic) en cette Province, lequel arrêt donne à l'Evêque diocésain, privativement à toutes autres personnes, la collation ou patronage des cures ou benéfices en ce pays.

5 Que le dit Demand ${ }^{r}$ ayant agrée cette nomination et accepté la collation de la dite cure ou benefice de la dite paroisse de $\mathbf{S}^{t}$ Jean Baptiste de Rouville, ainsi faite et octroyée en sa faveur par le dit Sieur Eveque de Quebec, en exécution des loix du Pays, en est par cela même devenu de fait et de plein droit, le seul curé et desservant légitime : qu'en cette qualité et capacité et a ce titre, le dit Demandeur s'est mis vers la fin d'Avril ou en Mai 1834, en possession réelle et légale de la dite cure, et bénéfice ainsi a lui conféré et alors devenu vacant comme dit est, et de leurs circonstances et dépendances: que depuis ce tems là il a toujours seul, comme curé de la dite paroisse de $\mathrm{S}^{\boldsymbol{t}}$ Jean Baptiste, possédé la dite cure ou benefice et leurs circonstances et dependances, et joui des droits, privileges, fruits et revenus $\mathbf{y}$ attachés, jusqu'à l'époque des troubles et vexations ci-après Mentionnées, et ce légalement, paisiblement, publiquement, et au vu et au Su de tout le Monde, et en particulier du dit Défendeur en cette cause.

6 Qu'outre cette possession légale, réelle et continue de la dite cure et bénéfice de la dite paroisse de $\mathrm{S}^{\mathbf{t}}$ Jean Baptiste pendant plusieurs années, ledit Demandeur a d'abondant, et pour mieux faire connaitre au public cette possession, ainsi que les droits et privilèges qu'il y avait acquis, et pour mieux en assurer la conservation à tous égards, fait le 3 Septembre dernier, acte solemnel, authentique et par écrit, de prise de possession civile de la dite cure et bénéfice, ce dont acte solemnel authentique et, légal a été rédigé et recu par écrit pardevant $\mathbf{M}^{\text {tre }}$ Charles Tétu et ses confrères notaires, le dit jour trois Septembre dernier, lequel acte de prise de possession civile a été duement insinué et enregitré (sic) dans le regitre (sic) des insinuations de cette cour, avec, en même tems, les Lettres, titres ou provisions susdites accordées et octroyées comme dit est par le dit Sieur Evêque de Quebec en faveur dud ${ }^{t}$ Demandeur.

7 Que la dite prise de possession civile a eu lieu en vertu et en conformité des loix en force en ce pays à cet égard.

8 Qu'en conséquence de tout ce que dessus, le dit Demd ${ }^{r}$, depuis led jour où il a été pourvu et nommé, comme curé, a la dite cure ou benefice de la dite paroisse de $\mathbf{S}^{t}$ Jean Baptiste, et quil a agrée et accepté cette nomination et collation, et qu'il s'est Mis en possession réelle et 
légale de la dite cure ou bénéfice, a été et est encore le seul curé de la dite paroisse, que comme tel, il a seul, jusqu'à l'époque des troubles et vexations ci-après mentionnés, possédé la dite cure et bénéfice, y a perçu la dime, y a exercé, en autant qu'il était en son pouvoir et comformément aux loix du pays, les devoirs \& fonctions de curé, a été comme tel de droit \& de fait le possesseur et le gardien du presbytère, de l'Eglise, sacristie et cimetière de la dite paroisse de $S^{t}$ Jean Baptiste, ainsi que de leurs circonstances et dépendances, et des droits \& privilèges y attachés, et ce à la connaissance et de l'aveu même du dit Défendeur.

9 Que du moment que led ${ }^{t}$ Demand $^{r}$ a été pourvu et nommé comme sus dit, comme curé, à la desserte de la dite cure ou bénéfice de la dite paroisse de $\mathbf{S}^{\mathbf{t}}$ Jean Baptiste, et quil a accepté cette collation ainsi faite en sa faveur par le dit Sieur Evêque de Quebec, et quil a eu pris comme dit est possession de la dite cure et bénéfice, il devait être, a été et est encore inamovible et perpétuel dans la possession de la dite cure et bénéfice, et des droits \& privilèges $\mathbf{y}$ attachés, et ce en vertu des lois en force en cette Province, et doit y être considéré et déclaré comme tel à tous égards : et qu'étant seul curé et desservant légitime de la dite cure et paroisse, aucun autre que lui ne peut y exercer légalement les fonctions curiales, ou sacerdotales et prendre le titre \& le nom de curé ou desservant de la dite paroisse, tant que le dit $\mathrm{Dem}^{\mathrm{r}}$ n'aura pas librement et de bon gré résigné la dite cure ou bénéfice, ou quil ne s'en sera pas démis librement \& volontairement, ou tant quil n'en aura pas été depossédé et exproprié par un jugement de cette cour, et que la dite cure ou bénéfice n'aura pas été déclarée vacante par un Jugement de la dite cour : et que jusqu'alors l'Evêque diocésain ne peut et n'a aucun droit, à moins de se rendre coupable d'infraction aux lois \& d'un abus flagrant de pouvoir, nommer un autre Ecclésiastique ou Prêtre a la desserte de la dite cure et bénéfice, soit sous le titre de curé ou sous tout autre titre.

10. Que depuis que le dit $\operatorname{Dem}^{r}$ a été ainsi pourvu et nommé à la dite cure ou bénéfice de la dite paroisse de $S^{t}$ Jean Baptiste de Rouville, le susdit Diocèse de Quebec a été demembré, et led ${ }^{\mathrm{t}}$ District de Montreal $a$ été érigé en un nouveau Diocèse, dont le dit Défendeur est Evêque en titre, sous les nom, titre et dignité d'Evêque de Montreal.

11. Que la dite cure ou bénéfice de la dite paroisse de $S^{t}$ Jean Baptiste de Rouville fait partie et dépend maintenant de ce nouveau Diocèse susdit, dont le dit Defendeur est comme dit est Evêque diocésain, et ce depuis l'existence même du dit nouveau Diocèse.

12 Et le dit Deman ${ }^{r}$ déclare de plus Que depuis qu'il a été nommé et pourvu, comme curé, a la desserte de la dite paroisse de $\mathrm{S}^{\mathbf{t}}$ Jean Baptiste de Rouville, et qu'il s'en est Mis en possession comme dit est, il a toujours rempli et exécuté, avec l'habileté, la diligence, la fidelité \& le soin possibles, tous les devoirs et toutes les obligations que lui imposait son ministère comme curé de la dite paroisse, tant sous le rapport religieux ou spirituel que sous le rapport civil, en Prêtre et citoyen également soumis aux lois de l'état Ecclésiastique et de la société dont il est membre, et ce à la parfaite connaissance et de l'aveu même de ses confrères et de ses Supérieurs Ecclésiastiques, et en particulier du dit Défendeur.

13 Que comme curé de la dite paroisse de $\mathrm{S}^{\mathrm{t}}$ Jean Baptiste, le dit $\operatorname{Dem}^{\mathbf{r}}$ est obligé de remplir non seulement des devoirs purement religieux ou spirituels envers les habitans qui composent cette paroisse, mais encore des devoirs \& des obligations que la loi civile lui impose tant envers eux qu'envers la Société entière, de même qu'aux autres curés en cette Province : et que c'est pour les mettre en état de remplir dignement ces devoirs et obligations que les loix du pays ont pourvu à leur subsistance, en leur affectant \& appropriant à leur usage exclusif, les oblations, dimes, fruits \& revenus des cures ou bénéfices dont ils sont pourvus. 
14 Que le dit Dem ${ }^{r}$ ayant embrassé l'Etat Ecclésiastique en devenant prêtre séculier dud ${ }^{t}$ Diocèse de Quebec, avec l'approbation $\&$ le consentement même de ses supérieurs Evêques, et notamment du dit Défendeur en cette cause, n'a pas d'autres Moyens de subsister que ceux qui peut fournir \& procurer, en cette Province, l'emploi de son ministère comme Prêtre, soit en qualité de curé ou autrement, desquels moyens de subsistance il devait s'attendre \& esperer de jouir sous la foi des loix de son pays, et desquels il ne peut être privé légalement par l'exercice arbitraire et abusif de l'autorité de ses Superieurs Evêques.

15 Que led $^{t} \operatorname{Dem}^{r}$, à la connaissance de ses supérieurs Ecclésiastiques, et notamment à celle du dit Défendeur, et de son propre aveu, a toujours été digne de desservir, comme curé, aucune cure ou bénéfice en cette Province, et notamment celle de la dite paroisse de $\mathbf{S}^{t}$ Jean Baptiste de Rouville.

16 Que quoique le dit Défen ${ }^{\mathrm{r}}$ ait eu et ait encore pleine et entière connaissance de tous les faits \& de toutes les circonstances ci-dessus mentionnés, et qu'il n'ait jamais eu et n'ait encore aucun reproche juste \& raisonnable a faire aud ${ }^{t}$ Dem $^{r}$ tant sous le rapport des Mours que sous le rapport de l'exercice de son Ministere \& de l'accomplissement de ses devoirs \& obligations comme Curé de la dite paroisse de $\mathrm{S}^{\mathrm{t}}$ Jean Baptiste, il a cependant le dit $\operatorname{Defen}^{r}$, tant avant que depuis qu'il a été revêtu des titre, qualité \& dignité d'Evêque de Montreal, \& qu'il est devenu ainsi Evếque en titre de ce dit Diocese de Montreal, et continuellement depuis ce tems, injustement, arbitrairement \& abusivement sévi contre le dit Demand ${ }^{r}$, sans cause juste et raisonnable, mais uniquement dans la vue et le dessein de ruiner le dit $\operatorname{Dem}^{\mathrm{r}}$ tant dans sa réputation que dans ses biens, de le disgracier, flétrir aux yeux du public \& de ses paroissiens : il a led ${ }^{t}$ Def, par suite des mêmes vues, desseins \& motifs injustes, arbitraires \& abusifs ci-dessus Mentionnés, tant avant le Mois de Septembre dernier, que dans tout le cours dud ${ }^{t}$ mois de Septembre dernier ainsi que dans le cours des Mois d'octobre \& Novembre aussi derniers, et continuellement depuis et jusqu'à ce jour, et toujours sans droit \& sans cause juste \& raisonnable, injurié, outragé, vexé et maltraité le dit Demand ${ }^{r}$, tant dans la dite Cité de Montreal que dans la dite paroisse de $S^{t}$ Jean Baptiste de Rouville, et ce tant verbalement que par écrit, actions \& voies de fait, ayant le dit Défendeur durant le tems ci-dessus mentionné $1^{\circ}$. dit, publié et écrit que le dit Demandeur était coupable de " l'acte le plus ouvert de révolte contre l'autorité de l'Eglise Sa Mère, de rebellion scandaleuse, de s'être souillé d'une tache publique de Mépris pour son supérieur, d'esclandre, et de plusieurs autres actes de même nature 》.

20. Chassé et Mis le dit $\operatorname{Dem}^{\mathbf{r}}$ hors de la sacristie de l'Eglise de $\mathrm{S}^{t}$ Jacques, en cette dite cité, et ce par vengeance, \& avec ignominie, en présence de plusieurs Prêtres assemblés, et dans la vue de le fletrir \& de le disgracier aux yeux de ces derniers et du public :

$3^{\circ}$. abusé, comme Evêque du dit Diocèse de Montreal, sans droit \& sans cause raisonnable, arbitrairement \& illégalement, du pouvoir que la loi lui donne de nommer aux cures vacantes en ce dit District pour déposséder led ${ }^{t} \operatorname{Dem}^{r}$ et l'exproprier abusivement \& illégalement, de la dite cure et bénéfice de la dite paroisse de $S^{t}$ Jean Baptiste, dont led $\operatorname{Dem}^{r}$ était \& est encore en possession \& dont il était et est encore le seul curé légitime comme dit est, en par le dit Défendeur le ou vers le 26 dud $^{t}$ mois de Septembre dernier nommant abusivement \& illegalement le dit Messire Pierre Lafrance pour desservir comme curé la dite paroisse de $\mathbf{S}^{\mathbf{t}}$ Jean Baptiste, et ce au Mépris des loix du pays \& des privilèges \& droits acquis $\operatorname{dud}^{t} \operatorname{Dem}^{r}$ et contre le consentement \& la volonté de ce dernier.

$4^{\circ}$. Abusé, comme Evêque dud ${ }^{t}$ Diocèse de Montreal, sans droit \& sans cause raisonnable, arbitrairement \& illégalement, de l'autorité \& des 
pouvoirs a lui conférés par la loi, pour autoriser led ${ }^{\mathbf{t}}$ Messire Pierre Lafrance \& lui permettre et enjoindre, comme en effet il l'a autorisé \& lui a permis et enjoint, au Mépris des loix du pays \& desd $^{\text {ts }}$ droits \& privileges acquis dud ${ }^{t}$ Dem $^{r}$, de celebrer la Messe et exercer les autres fonctions curiales dans la dite paroisse de $\mathrm{S}^{\mathbf{t}}$ Jean Baptiste de Rouville, quoique led $^{t}$ Dem $^{r}$ fut alors, comme il est encore, le seul cure legitime de la dite paroisse.

$5^{\circ}$. Avisé, conseillé, et ordonné au dit Messire Pierre Lafrance, et a plusieurs autres personnes, abusivement, illégalement \& au Mépris des loix du Pays et de l'ordre public, de s'emparer \& de prendre possession, par force, violences, \& voies de fait comme en effet led ${ }^{t}$ Messire Pierre Lafrance \& ces dites autres personnes, par suite de ces conseils \& de ces ordres illegaux dud ${ }^{\mathbf{t}}$ Def, se sont emparés et ont pris possession, par force, violences \& voies de fait, et meme à Main armée des dites Eglise, sacristie \& Cimetière de la dite paroisse de $S^{t}$ Jean Baptiste, dont led Dem $^{\mathrm{r}}$ était en possession \& gardien comme dit est, pour déposséder et exproprier de fait le dit Deman ${ }^{\mathrm{r}}$, de sa dite cure \& bénéfice, \& le priver des droits \& privilèges quil y avait acquis, \& l'empêcher d'y remplir les devoirs de son ministère.

$6^{\circ}$. enjoint \& ordonné aud ${ }^{t}$ Messire Pierre Lafrance, abusivement \& illégalement, de percevoir les dimes et oblations de la dite cure et bénéfice de la dite paroisse de $S^{t}$ Jean Baptiste, et ce au Mépris des lois du pays, et desd ${ }^{\text {ts }}$ droits acquis dud ${ }^{t}$ Dem $^{\mathbf{r}}$. pour en priver et dépouiller ce dernier, et lui oter injustement \& illégalement tous ses Moyens de subsistance, \& le Mettre hors d'état de remplir les devoirs de son Ministère.

$7^{\circ}$. Abusé, comme Evêque dud ${ }^{t}$ Diocèse de Montreal, sans droit \& sans cause raisonnable, arbitrairement \& illégalement, de l'autorité des pouvoirs à lui conférés par la loi, pour oter aud ${ }^{t} \operatorname{Dem}^{r}$ toute jurisdiction dans la dite paroisse de $\mathrm{S}^{\mathbf{t}}$ Jean Baptiste, \& le suspendre de l'exercice de ses fonctions curiales en la dite paroisse, \& déclarer \& jurer qu'a compter du 27 dud $^{\mathbf{t}}$ mois de Septembre dernier, le dit $\operatorname{Dem}^{\mathrm{r}}$, ne serait plus approuvé pour le ministere dans aucune partie de son diocèse savoir le dit Diocèse de Montreal, ainsi qu'il appert par une lettre ou ordonnance dud ${ }^{t}$ Défendeur, en date du 14 Sept : dernier et par lui écrite, signée et adressée aud ${ }^{t} \operatorname{Dem}^{r}$ et ce dans la vue et le dessein de ruiner, flétrir \& disgracier le dit Demand ${ }^{\mathrm{r}}$.

$8^{\circ}$. Abusé, comme Evêque du dit Diocèse de Montréal, sans droit, arbitrairement de l'autorité \& des pouvoirs a lui conférés par les loix du pays, pour créer, comme en effet il a crée \& érigé au mépris de ces memes loix, un espèce de tribunal devant lequel il a pris sur lui, abusivement, arbitrairement \& illégalement, de citer $\operatorname{led}^{t}{ }^{\mathrm{Dem}} \mathrm{d}^{\mathrm{r}}$, pour le disgracier le fétrir, l'outrager \& le vexer sous prétexte de l'obliger à répondre à de pretendus accusations, ainsi qu'il appert par une lettre ou ordonnance dud ${ }^{t}$ Defend $^{r}$, en date du 25 oct : dernier, et par lui écrite, signée, et adressée au dit Demd ${ }^{\mathbf{r}}$ : Et ensuite prononcé, publié, le ou vers le 3 novembre dernier, une sentence frappant le dit Demand. d'interdit, et le déclarant, jusqu'à nouvel ordre de sa part, suspens de toutes fonctions sacerdotales ou cléricales, et réduit à la communion laique, laquelle sentence il a fait signifier aud ${ }^{t}$ Demd $^{r}$.

17. Et le dit Demandeur déclare que tous les faits ci-dessus Mentionnés pour lesquels il reclame contre le dit Défendeur, ont eu lieu tant dans la dite cité de Montreal que dans la dite paroisse de $\mathbf{S}^{\mathbf{t}}$ Jean Baptiste de Rouville respectivement, et ce tant dans le cour dud mois de Septembre dernier que dans le cours des dits Mois doctobre \& de Novembre aussi derniers, et continuellement durant ces diverses époques et depuis jusqu'à ce jour.

18 Que le dit Défendeur, en se conduisant envers le $d^{t}$ Demandr. de la manière qui vient d'être rapportée, non seulement est coupable à son 
égard d'un abus flagrant de pouvoir, mais encore il a agi, dans cette conduite, injustement, sans aucun droit ni raison quelconque, arbitrairement, illégalement, a foulé au pied toutes les loix du pays, pour flétrir, vexer et ruiner, dans la personne du Demand ${ }^{x}$, un inférieur qui a toujours fidelement rempli ses devoirs religieux et civils, a été constam. ment soumis aux ordres justes \& raisonnables de ses supérieures Ecclésiastiques et aux loix de son pays, sans renoncer toutefois au droit \& privilège qu'a tout citoyen d'invoquer l'appui de ces mêmes loix, et à qui led $^{t}$ Defend $^{r}$ lui-même, n'a rien à reprocher ni sous le rapport des mœurs ni sous le rapport de l'exercice de son ministère.

19. Que par cette conduite arbitraire, vexatoire, abusive \& illégale de la part dud ${ }^{t}$ Defendeur, led ${ }^{t}$ Demd $^{r}$ se trouve privé du pouvoir et des moyens de remplir, envers les paroissiens de la dite paroisse de $\mathbf{S}^{\mathbf{t}}$ Jean Baptiste dont il a toujours été et est encore le seul curé légitime, ainsi qu'envers la Société entière, les devoirs tant religieux que civils que, comme curé de la dite paroisse, il est obligé de remplir, et se trouve de plus injustement disgracié, flétri \& deshonoré dans l'esprit de ses concitoyens sans l'avoir Mérité, et reduit a la misère la plus complète.

20. Que le dit Demand, dans des vues de paix \& de conciliation, \& quoique fort de la justice de ses droits \& de sa position, a cependant, en autant que les circonstances pouvaient le permettre, fait faire, par l'entremise de quelques-uns de ses confrères membres du clergé, des démarches respectueuses auprès du dit Défendeur, pour l'engager à revenir à son égard à des sentimens plus doux et plus conformes à la justice, et à rapporter tous ses actes arbitraires \& illegaux comme susdit, mais toujours en vain; de sorte qu'il ne reste plus aud ${ }^{\mathbf{t}}$ Demd $^{r}$ d'autre recours que celui de s'adresser aux tribunaux competens pour obtenir la justice qui lui est due.

21. Que par cette conduite de sa part led ${ }^{t}$ Defend $^{r}$ a fait en outre éprouver et fait éprouver tous les jours aud ${ }^{t} \operatorname{Demd}^{\mathbf{r}}$ des dommages considerables, lesquels dommages il parte et estime à la somme de L 2000 cours actuel qu'il a ainsi droit, pour les raisons susmentionnées, de repéter et recouvrer dud ${ }^{t}$ Défendeur.

Pourquoi led $d^{t}$ Demandeur conclut à ce que, pour les raisons, causes et considérations ci-dessus mentionnées, les provisions, Lettres ou titres qui ont été accordées par led ${ }^{t}$ Defend $^{r}$ au dit Messire Pierre Lafrance, pour desservir, au préjudice dud ${ }^{t}$ Demand ${ }^{\mathrm{r}}$. la dite cure ou bénéfice de la dite paroisse de $\mathrm{S}^{\mathbf{t}}$ Jean Baptiste de Rouville, la dite lettre ou ordonnance dud ${ }^{t}$ Défendeur du 14. Septembre dernier, celle susdite du même en forme de citation du 25 octobre aussi dernier, et la dite sentence du 3 Novembre aussi dernier, ensemble, ce qui les a suivies et précédées, soient à tous égards déclarées nulles, abusives et vexatoires, et contraires à la justice \& aux loix du pays : que lui le dit Demandeur soit maintenu et gardé en la possession et jouissance de la dite cure ou bénéfice \& des dites Eglise, sacristie presbytère \& cimetière, ainsi que des droits, privilèges, fruits et revenus y attachés, de laquelle cure ou bénéfice le dit Demandeur a été comme dit est canoniquement \& legalement pourvu, \& qu'il y soit déclaré inamovible et perpetuel au désir des lois du pays : qu'il soit fait défenses au dit Défend ${ }^{r}$ de troubler le dit Demand ${ }^{r}$ en la dite possession et jouissance, en quelque maniere que ce soit : et qu'enfin le dit Défendeur soit condamné en outre à payer au dit Demandeur, par forme de dommages comme susdit, la dite somme de $L 2000$ cours actuel : le tout avec intéret $\&$ dépens.

Montreal 27 Janvier 1837. 
Messire Louis Nau Prêtre et Curé de la paroisse de Saint Jean Baptiste de Rouville dans le district de Montréal, et résident en la dite paroisse

Demandeur

vs

Sa Grandeur Monseigneur Jean Jacques Lartigue Evêque de Montréal résidant en la Cité de Montréal dans le dit district,

Le $1^{\text {er }}$ fevrier 1837

Défendeur

Le Sherif fait le retour du Writ et de la declaration en Cette Cause. deur.

Messieurs La Fontaine et Berthelot Comparaissent pour le deman-

Le défendeur appellé fait défaut.

La cour sur motion du demandeur ordonne que les Exhibits Numeros un, deux, trois, quatre, cinq, Six, Sept, huit, neuf, dix, onze. douze et treize par lui produits dans une Cause $N^{\bullet} 500$, dans laquelle il est demandeur contre Messire Pierre Lafrance soit regardés et Considérés Comme produits et filés en cette Cause sous les mêmes numéros.

Le demandeur file une liste et vingt deux Exhibits.

Le $4^{\circ}$ février 1837.

La Cour sur motion du défendeur lui permet sous toutes réserves de droit de lever le défaut entré... contre lui le premier du courant, et de Comparaître... également sous toutes réserves de droit par Messieurs Peltier \& Bourret, ses avocats.

\section{Le $6^{\bullet}$ février 1837.}

La Cour sur motion du defendeur et du Consentement du demandeur ordonne que les Exhibits Numéros un, deux, trois et quatre filés par Messire Pierre Lafrance dans une Cause $\mathrm{N}^{\circ} .500$. ou le demandeur est demandeur contre le dit Messire Pierre Lafrance et actuellement pendante devant Cette Cour soient considérós comme produits et filés par le dit défendeur au Soutien de l'exception... Déclinatoire par lui plaidée en cette Cause et comme faisant partie de la procédure en icelle.

Le défendeur file une liste, exception déclinatoire et quatre exhibits.

$$
\text { Le } 8^{\circ} \text { fevrier } 1837 .
$$

La Cour sur motion du defendeur et du consentement du demandeur ordonne que les Exhibits Numéros Cinq, Six et Sept produits et filés par Messire Pierre Lafrance dans une Cause $N^{\circ} 500$ : où le dit demandeur est demandeur contre le dit Messire Pierre Lafrance et actuellement pendante devant Cette Cour soient considérés comme produits et filés par le dit défendeur en cette Cause au Soutien des défenses au fonds en droit, Exceptions péremptoires et fins de non recevoir et défenses par lui plaidées et comme faisant partie de la procédure en la présente Cause.

Le defendeur file une liste, défenses au fonds en droit, exceptions péremptoires et fins de non recevoir et défenses et Sept Exhibits.

$$
\text { Le } 1^{\text {er }} \text { Mai } 1837 .
$$
défenses.

Le demandeur file Reponses aux exceptions et Repliques aux

$$
\text { Le } 1^{\text {or }} \text { Juin } 1837 .
$$

Le demandeur inscrit cette Cause sur le role de droit pour argument en droit le Cinquième Jour de Juin Courant. 


\section{Le $5^{\circ}$ Juin 1837.}

La Cour Continue la règle qui fixoit Cette Cause pour argument ce jourd'hui au Sept du Courant.

\section{Le $7^{\circ}$ Juin 1837.}

Les parties ont été entendues

$$
\text { C. A. V. }
$$

Les parties en Cette Cause par leurs avocats et procureurs consentent a ce que la Cour pour décider Sur l'audition en droit en icelle prenne Connaissance des pièces ou exhibits produits par les parties respectivement, et qu'à cet effet elles les regarde comme authentiques; les dites parties se reservant néanmoins le droit de produire plus tard à l'appui de leurs allégués, que la Cour ne regardera pas comme prouvés, telles autres preuves légales quelle jugeront à propos.

$$
\mathrm{N}^{\circ} \mathbf{5 0 0} \text {. }
$$

Messire Louis Nau, prêtre et Curé de la paroisse de $\mathbf{S}^{t}$ Jean Baptiste de Rouville, dans le district de Montréal, et résident en la dite paroisse,

Demandeur

vs

Messire Pierre Lafrance, prêtre, Cidevant résident en la paroisse de la Rivière des Prairies, dans le dit district, et maintenant résident en la dite paroisse de $\mathbf{S}^{\mathbf{t}}$. Jean Baptiste de Rouville

\section{Le $1^{\text {ex }}$ Février 1837.}

Défendeur

Le Sherif fait le retour du writ et de la declaration en Cette Cause.

Messieurs La Fontaine et Berthelot Comparaissent pour le demandeur.

Le defendeur appellé fait défaut.

Le demandeur file une liste et treize exhibits.

Le 4 février 1837 .

La Cour sur motion du defendeur lui permet sous toutes réserves de droit de lever le défault entré contre lui le premier du Courant, et de Comparaitre également sous toutes réserves de droit par Messieurs. Peltier \& Brunet ses avocats.

\section{Le $6^{\circ}$ février 1837.}

Le défendeur file une liste, exception déclinatoire et quatre Exhibits.

$$
\text { Le } 8^{\circ} \text { février } 1837 .
$$

Le defendeur file une liste, dépenses au fonds en droit, exceptions péremptoires et fins de non recevoir et défenses, et sept Exhibits.

$$
\text { Le } 1^{\text {er }} \text { Mai } 1837 .
$$

Le demandeur file réponses aux exceptions et répliques aux défenses.

$$
\text { Le } 1^{\text {er }} \text {. Juin } 1837 .
$$

Le demandeur inscrit cette Cause sur le role de droit pour argument. en droit le cinq du Courant. 
Le $5^{\circ}$ Juin 1837.

La Cour Continue la règle qui fixoit cette Cause pour être entendu en droit à ce jourd'hui, au six du Courant.

Le $6^{\circ}$ Juin 1837.

La Cour Continue a demain pour entendre les parties en droit sur la regle du premier du Courant.

Le $7^{\circ}$ : Juin 1838.

Les : parties ont été entendues en droit.

C. A. V.

Les parties en cette Cause par leurs avocats et procureurs Consentent par les présentes à ce que la Cour pour décider sur l'audition en droit en icelle prenne Connaissance des pièces ou exhibits produits par les parties respectivement, et qu'à cet effet elle les regarde Comme authentiques; les dites parties se réservant néanmoins le droit de produire plus tard à l'appuie de leurs allégués que la Cour ne regardera pas Comme prouvés telles autres preuves légales qu'elles Jugeront à propos ${ }^{35}$.

$\mathrm{M}^{\mathrm{gr}}$ Lartigue mandait en janvier 1837 à $\mathbf{M}^{\mathrm{gr}}$ Turgeon que $\mathrm{M}$. Nau avait soulevé tout le monde contre lui par la violence et l'injustice de ses procédés. Et en février suivant, il signalait à l'archevêque, $M^{\text {Bx }}$ Signay, que la dispute est portée devant les tribunaux, mais que - selon la louable habitude des avocats, deux des procès ne viendront pas avant avril et les deux autres avant septembre ${ }^{36}$ ».

C'est la première nouvelle que j'aie de ces deux autres procès. Mais l'évêque avait mal présumé, pour une fois, de la célérité des tribunaux. En mars 1837 , écrivant à nouveau à $M^{\text {gr }}$ Turgeon, il lui apprend que le curé de Saint-Jean-Baptiste [M. Lafrance] et ses six coaccusés ont été complètement acquittés par la Cour criminelle ${ }^{37}$.

Des ecclésiastiques qui s'accusent en Cour pénale, c'est à tout le moins inattendu.

Le même jour, écrivant à l'archevêque $\mathrm{M}^{\mathrm{gr}}$ Signay, « après la perte de son procès [en Cour pénale] par M. Nau, on ne sait, disait-il, que pensent les juges relativement aux curés inamovibles ${ }^{38}$.

Communiquant, en septembre 1837, avec l'évêque coadjuteur de Québec, $\mathbf{M}^{\mathrm{gr}}$ Lartigue déclarait que l'esclandre de $M$. Nau a accoutumé d'autres' prêtres $\mathrm{du}$ diocèse à la résistance ${ }^{39}$. On pouvait 's'y attendre...

En mars précédent, il avait fait observer à M. Ducharme, de SainteThérèse-de-Blainville : « Par suite de la révolte de $\mathrm{M}$. Nau, il voit, encore plus que lui, les malheurs qui menacent le diocèse par la mauvaise conduite et l'esprit d'indépendance qui règne dans le clergé ${ }^{40}$.

36 R.A.P.Q. pour 1944-1945, p. 231.

37 R.A.P.Q., passim, p. 235.

38 R,A.P.Q, passim, p. 233.

39 R.A.P.Q., passim, p. 250.

40 R.A.P.Q., passim, p. 236. 
En octobre 1837, il interdisait au curé Lafrance, de Saint-JeanBaptiste, pourtant nommé par lui, de se loger dans le presbytère, jusqu'à nouvel ordre ${ }^{41}$.

Dès fin novembre 1837, M. Nau avait quitté Saint-Jean-Baptiste et demandé asile au curé de Sainte-Anne-du-Bout-de-l'Ile, M. de Lamothe, un Français venu au pays en 1813 comme aumônier du régiment de Watteville, et qui après avoir été missionnaire au Haut-Canada, fut le premier curé de Sainte-Scholastique (Deux-Montagnes). L'évêque lui disait « souhaiter que ceux qui donneront asile à M. Nau montrent qu'ils sont éloignés de ces mauvais principes et de ceux de son avocat - une flèche à La Fontaine! - et qu'ils puissent le faire rentrer en luimême ${ }^{42}$ ".

Les terribles événements de novembre et décembre 1837 allaient quelque peu faire oublier l'affaire Nau.

C'est dans une lettre du 28 décembre au cardinal-préfet de la Propagande à Rome, que la question de droit avait été posée par l'évêque de Montréal.

Quant aux cures diocésaines, un décret de Mgr Laval, du 26 mars 1663, approuvé par le Roi le mois suivant, établit qu'elles seraient toujours révocables au gré de l'Evêque, et si les Evêques en rendirent quelques-unes inamovibles sous le régime français, il n'en fut pas ainsi depuis la conquête anglaise, excepté pour les cures de Québec et de Montréal.

Il est vrai que le Roi de France, en 1679, émit un nouveau décret pour rendre les cures inamovibles, mais ce fut à l'insu de l'Evêque, qui continua de nommer dans les paroisses des curés révocables à volonté, et depuis plus de soixante-dix ans pas une seule cure inamovible n'a été érigée, ni même d'autres, jusqu'à ces dernières années, et il n'y a pas d'officialité pour corriger ou déposer les clercs indignes, parce que notre gouvernement hétérodoxe refuserait d'en reconnaître l'autorité. Récem. ment, toutefois, Louis Nau, nommé comme curé révocable de Saint-JeanBaptiste de Rouville, puis démis de sa mission par l'Evêque de Québec, prit possession de la paroisse par-devant des notaires laics, et bien que suspendu de ses fonctions sacerdotales, empêcha son successeur d'exercer ses droits curiaux en s'adressant à une Cour civile.

Aussi serait-il avantageux d'avoir de Rome un décret pour confirmer l'édit Episcopal de 1663 et déclarer l'amovibilité de tous les curés en ce pays, excepté le premier curé de Québec ou celui de Montréal 43 ?

$M^{g r}$ Lartigue croyait devoir dire, en avril 1838, à $M^{\mathrm{gr}}$ Provencher, de la Rivière-Rouge, que "les juges hésitent à se prononcer sur le procès $\mathrm{Nau}^{44}$.

Edmond Lareau, précité, a consigné que le décret fut rendu par les juges Reid [James], Pyke [George] et Rolland [Jean-Roch].

41 R.A.P.Q., passim, p. 252.

42 R.A.P.Q., passim, p. 259.

43 R.A.P.Q., passim, p. 227.

44 R.A.P.Q. pour 1945-1946, p. 56. 
Citons au texte cette sentence fameuse :

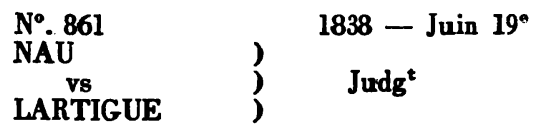

La Cour apres avoir entendu les parties par leurs avocats, examiné la procedure et les pieces produites comme preuves, rendues autentiques par le consentement des parties, et sur le tout délibéré - Se declare incompétente à prendre connoissance sur la presente demande de la sentence rendue par le Defendeur en sa qualité d'Evêque Diocesain, qui suspend le Demandeur de ses fonctions sacerdotales ou Curiales, et de la procedure qui a eu lieu devant le Tribunal de l'Evêque à cet egard Et faisant droit sur les autres conclusions de la demande, considérant que le Demand ${ }^{\text {n'a }}$ eu ni titre, ni possession, comme Curé en titre de la Cure et paroisse de St Jean Bap ${ }^{\text {to }}$ de Rouville, de manière à pouvoir reclamer le Bénéfice de cette Cure, Que la lettre de Mission à lui accordée pour la desserte de la dite paroisse, a été revoquée, comme elle pouvoit l'être par le Défendeur, Evêque actuel de cette partie du ci-devant Diocèse de Quebec, et que les prétentions du Demandeur étant d'ailleurs malfondées - $\mathrm{A}$ debouté et deboute le Demand ${ }^{\boldsymbol{r}}$ de son action avec depens ${ }^{45}$.

Le jugement inscrit au registre du tribunal n'est pas signé, à la mode du temps, comme aujourd'hui encore en certains tribunaux inférieurs.

Certes, $M^{g r}$ Lartigue eut raison d'en être content et il s'en ouvrit le même mois à l'évêque coadjuteur de Québec, $M^{\mathrm{gr}}$ Turgeon : - Le jugement contre $M$. Nau lui a paru aussi bien motivé qu'on pouvait l'attendre d'un (?) juge protestant ${ }^{46}$."

Ce procès nous valut au moins l'un des rares écrits qui nous soient parvenus de sir Louis-Hippolyte La Fontaine, soit sa brochure Notes sur l'Inamovibilité des Curés dans le Bas-Canada ${ }^{47}$, auquel ouvrage l'évêque Lartigue aurait répondu, ou fait répondre, par un Mémoire sur l'Amovibilité des Curés en Canada ${ }^{48}$.

En septembre 1838, l'évêque de Montréal prévenait le successeur de Nau à Saint-Jean-Baptiste, M. Lafrance, qu'il avait reçu de celui-là un avis d'inscription en appel. Il ajoutait, non sans malice : on couperait l'appel dans sa racine en prouvant que les deux cautions, Charles Têtu, notaire, et Hubert Raynaud-Blanchard ne sont pas capables ensemble de répondre des frais des deux procès. Il conseillait au curé d'engager la Fabrique en assemblée à soutenir l'appel et à payer les frais comme au premier procès ${ }^{49}$.

$\mathrm{M}^{\mathrm{gr}}$ Lartigue avait retenu comme conseils son cousin, CômeSéraphin Cherrier et Charles Mondelet, de Montréal, et René-Edouard

45 Registre de la Cour... Voir note 35.

46 R.A.P.Q. pour 1945-1946, p. 66.

47 Montréal, Imprimerie de la Minerve, 1837. Exemplaire à la Bibliothèque

Saint-Sulpice de Montréal.

48 R.A.P.Q. pour 1944-1945, p. 237.

49 R.A.P.Q. pour 1945-1946, p. 79. 
Caron, de Québec, qui fut le premier maire de sa ville et mourut lieutenant-gouverneur de la province.

Nau lui-même avait pris comme procureur - était-ce en l'absence de Louis-Hippolyte La Fontaine, passé en Europe au lendemain de l'Insurrection ? - James Stuart, à qui fut subrogé J. O'Kill Stuart, son parent, lorsque James Stuart devint juge en chef de la Cour du banc du roi en 1838 .

Cependant $\mathrm{M}^{\mathrm{gr}}$ Bourget nous apprend, en avril 1839, que La Fontaine était toujours l'avocat principal de M. Nau, bien que Stuart apparût à l'inscription en appel ${ }^{51}$.

Il devait être encore beaucoup question dans la correspondance de $M^{3 r}$ Lartigue de l'appel du curé Nau à la Cour de Québec, dont la dernière fois, en janvier 1840, trois mois avant son décès ${ }^{52}$.

$M^{\text {gr }}$ Lartigue avait convié $M$. Nau, en août 1839 , à la retraite générale du clergé, tenue au petit séminaire de Montréal ${ }^{53}$.

En avril 1840, au décès de $M^{\mathrm{gr}}$ Lartigue, $\mathrm{M}^{\mathrm{gr}}$ Bourget demandait au coadjuteur de Québec, $M^{\text {r }}$ Turgeon, de faire remettre l'appel de M. Nau, en attendant la loi nouvelle de judicature ${ }^{54}$.

Et j'ignore encore si jamais, après tant de tergiversations, jugement fut rendu en appel.

$M^{\text {gr }}$ Bourget invitait, en janvier 1841, M. Nau, prêtre à Saint-JeanBaptiste, ne pouvant aller le voir pour le tirer \& du malheureux état où vous vivez depuis quelques années », à venir le rencontrer au plus tôt ${ }^{55}$.

$M^{g r}$ Bourget écrivait en février 1842, à $M^{g r}$ Turgeon, coadjuteur de Québec, qu'il croyait M. Nau l'auteur de l'article de l'Aurore, signé Un Curé, paru à propos de manifeste de M. Etienne Chartier.

Le mois suivant, l'évêque de Montréal [Bourget] faisait part à son archevêque [Signay] qu'il lui enverra un exemplaire des Observations de M. Tétu sur \& vos droits et les miens », publication qui ajoute au * scandale, déjà assez grand ", donné par M. Nau.

En juillet suivant (1842) il disait au nouveau curé de Saint-JeanBaptiste, M. J.-B. Dupuy, se réjouir de la conversion de M. Nau, * qui ne doit rien craindre de l'indignation de l'évêque mais qui ne pourra être placé dans le ministère qu'après avoir satisfait à tout ce qu'exigent les règles de l'Eglise et les saints Canons ${ }^{56}$ s.

50 R.A.P.Q., passim, p. 82

51 R.A.P.Q, passim, p. 195.

52 R.A.P.Q., passim, p. 124.

s3 R.A.P.Q., passim, p. 110.

54 R.A.P.Q., passim, p. 224.

55 R.A.P.Q. pour 1946-1947, p. 130.

56 Renseignements du R.P. Iéon Pouliot, s.j. (Archives de l'archevêché de Montréal). Les remerciements de l'auteur sont acquis au chancelier de l'archevêché de Montréal, M ${ }^{g r}$ G.-Robert Mitchell, P.D., et à ses próposés aux Archives pour la bienveillance de leur accueil à l'été 1957. 
Le prélat avait-il présumé trop tôt de l'action de la grâce ?

Dès le mois d'août, il devait se raviser et prier M. Crevier, de SaintHyacinthe, d'avertir les fidèles des missions des townships (sic) où M. Nau a osé entendre les confessions, que « ce prêtre intrus ne peut ni prêcher, ni confesser, ni dire la messe, ni exercer aucune fonction ecclésiastique 》.

Enfin, mai 1843, $\mathrm{M}^{\mathrm{gr}}$ Bourget remerciait $\mathrm{M}^{\mathrm{gr}}$ Patrick Phelan, vicaire général à Bytown [Ottawa] de "la charité exercée envers M. Nau * et formait le vœu qu'il fasse pénitence du passé, qu'il rétracte publiquement ses écrits erronés et promette de mieux faire à l'avenir.

Et oncques n'entendîmes depuis parler de M. Louis Nau, curé, peu heureux, de Sainte-Madeleine de Rigaud et de Saint-Jean-Baptiste de Rouville aux temps troublés de 1830 .

Jean-Jacques LeFEBvRE, M.S.R.C., Archiviste, Palais de Justice, Montréal 